\title{
Agents with Emotional Intelligence for Storytelling
}

\author{
João Dias, Ana Paiva INESC-ID \\ Av. Prof. Cavaco Silva, TagusPark \\ 2780-990 Porto Salvo, Portugal \\ joao.dias@gaips.inesc-id.pt, ana.paiva@inesc-id.pt
}

\section{INTRODUCTION}

In trying to build increasingly believable autonomous characters for virtual environments, researchers successfully explored the use of emotional systems to endow their agents with emotional capabilities. Of relevant notice are the ability to experience and express emotions, and the ability to use its emotions to influence decision-making. These systems are often based in appraisal theories, such as OCC [5], which claim that emotions are a result of subjective evaluations (appraisals) of events and situations.

However, agents with full Emotional Intelligence have been largely unaddressed so far. By Emotional Intelligence we mean the definition proposed by Salovey and Mayer[6, 4]:

"Emotional intelligence refers to an ability to recognize the meanings of emotion and their relationships, and to reason and problem-solve on the basis of them."

Although the ability to monitor feelings and emotions has been addressed, having explicit knowledge about the appraisal process and other's emotions, and using that knowledge to reason about emotions and build plans of actions, has not. This is due in part to the fact that one cannot address this problem without first tackling the other components. Only now we are ready to start addressing it.

We believe that Emotional Intelligence is an important component to achieve more human-like and believable behaviour, especially in a Storytelling scenario where social interaction and emotional conflicts take a major role. It is true that it is still possible to achieve believable behaviour without this component in a storytelling scenario (as in FearNot![2]), because we can author the characters in a way to portray such emotional intelligence. However, when facing an interacting user, this is much harder to do without a complete Emotional Intelligence. Thus, Emotional Intelligence offer us more flexibility in achieving believable social behaviour.

\section{RELATED WORK}

Continuing their research in Emotional Intelligence, Mayer and Salovey put forward a four branch model that divides Emotional Intelligence in four main skills:

- perceiving emotions in oneself and others - has to do with the perception and expression of emotion through gestures, facial expressions, or other communication mechanisms. This area was the first one to be addressed by researchers, and is still an important subject of research in IVAs and ILEs.
- using emotions to facilitate thought - the second most researched skill, focus on using emotions to guide cognitive processes, such as learning and adaptation, attention and decision making.

- understanding emotions - the idea here is that emotions convey information. For instance, Anger indicates a possible intention of harming other. Therefore, understanding emotions involves understanding the meaning of emotions, together with the capacity to reason about those meanings. This skill together will be the focus of our work.

- managing emotions - once a person understand emotions, it can manage one's own and other's emotions in order to promote social goals. For instance, one can go see a movie when distressed in order to feel better, or do something pleasant to help a friend come out of a bad mood. To some extent, this skill was addressed by the work of Marsella and Gratch in EMA[3], where they apply emotion coping strategies to deal with one's negative emotions.

\section{MODEL}

The proposed model will be integrated and implemented in an existing emotional agent architecture, named FAtiMA[2, 1]. In FAtiMA, emotions result from a subjective appraisal of events according with OCC Theory. The architecture is divided in two main layers, a reactive and a deliberative one. The first layer is responsible for the agent's reactive behaviour and is composed by: a set of emotional reaction rules that define OCC's appraisal variables such as desirability which are then used to generate emotions; and by a set of action tendencies (AT) that represents the character's impulsive actions (e.g. crying when very distressed). The deliberative layer is responsible for the agent's goaloriented behaviour and means-ends-reasoning. It also has an appraisal component that generates emotions from the state of plans in memory. The Knowledge Base and the Autobiographic Memory are the main memory components. The top of Figure 1 shows a simplified diagram of the architecture.

In order to extend FAtiMA with the ability to understand emotions, we must first endow the planner with explicit knowledge about the Appraisal Process. This can be done, by translating the emotional reaction rules into planning operators, which use a STRIPS notation. Then, the OCC rules used to create emotions from the appraisal variables must also modeled as planning operators. For instance, the rule 


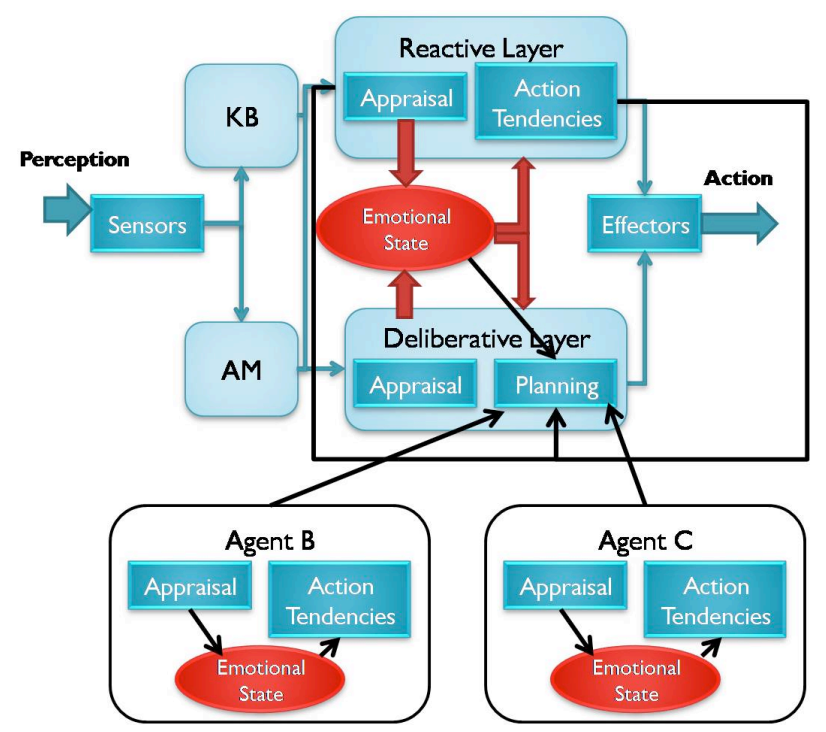

Figure 1: Extending FAtiMA with the skill of understanding emotions

that maps an undesirable event into the emotion of distress is translated into an operator which has the precondition that an event is undesirable and has the effect of causing distress. Additionally to the Appraisal Process, is also important to model actions that are triggered from particular emotional states, i.e. Action Tendencies. The translation is achieved by modeling action tendencies as operators where the preconditions correspond to the emotional state that triggers the AT. Finally, the planner must also have knowledge about the agent's emotional state. These connections are represented in Figure 1 by the black arrows coming out of the appraisal and AT processes.

This first step gives our agent the capability to reason about his own's emotions but only partially about other's emotions. Although it is true that the agent can assume that others are like him and use his information to predict how others will feel, this will often lead to wrong assessments. Given the subjective nature of appraisal, the agent must build a model of how others appraise events and react to a given emotion. So, if the agent A knows two other agents $\mathrm{B}$ and $\mathrm{C}$ he will, additionally to its own structures it will model the other agents' emotional reaction rules, emotional state and action tendencies (as seen in bottom of Figure 1). Initially, when the agent first meets another agent he will start with a model equal to his own (he assumes that others are like him). But as time goes by, the agent will refine the model it has about that new agent. For instance, if a given event is thought to be undesirable to another agent, but that agent happens to express joy or happiness, the agent will have to update the desirability value for that event.

\section{ILLUSTRATIVE EXAMPLE}

We will give a brief example of what kind of reasoning an agent can do with this emotional information. In order to model a bullying scenario, we can model a bully character with a high level goal of making the victim cry. In order to achieve this goal, the bully knows that the victim cries when it's very distressed (an AT), so the planner will try to force the victim to become distressed. The planner will also know that distress is caused by an undesirable event, and will consider all actions undesirable for the victim (kicking, pushing, insulting, etc). The deliberative layer will then select one of the alternatives and execute it. If everything goes as planned the bully will succeed and become satisfied. However, if the victim doesn't cry but seems happy instead, he will either try something else or eventually fail to bully the victim.

\section{FINAL CONSIDERATIONS}

The proposed extensions will have strong implications in some of the core components of the architecture. In the current architecture there is an initial deliberation where a goal is selected, and then the means-ends reasoning takes full control of the rest. With the proposed model, there will be several levels of deliberation and commitment, interleaved with planning. Moreover, by modeling behaviour with higher-level goals, which can expand to a wide number of alternative solutions, we will increase the search space and planning may become intracktable. We believe that this problem can be solved by using emotional information as a heuristic to guide and constrain means-ends reasoning, which actually corresponds to the second skill in Mayer's model. Thus, on an ending note, we point out that in order to tackle the last two skills and build agents with Emotional Intelligence we need to address all the four skills.

\section{ACKNOWLEDGEMENTS}

This work was supported by a scholarship (SFRH BD/19481/2004) granted by the Fundação para a Ciência e a Tecnologia (FCT) The authors are solely responsible for the content of this publication. It does not represent the opinion of the FCT, which are not responsible for any use that might be made of data appearing therein.

\section{REFERENCES}

[1] J. Dias. Fearnot!: Creating emotional autonomous synthetic characters for empathic interactions. Master's thesis, Universidade Técnica de Lisboa, Instituto Superior Técnico, Lisboa, 2005.

[2] J. Dias and A. Paiva. Feeling and reasoning: a computational model for emotional agents. In Proceedings of 12th Portuguese Conference on Artificial Intelligence, EPIA 2005, pages 127-140. Springer, 2005.

[3] G. J. and M. S. A domain-independent framework for modeling emotion. Cognitive Systems Research, Volume $5(4), 2004$.

[4] M. J., C. D., and P. Salovey. Emotional intelligence meets traditional standards for an intelligence. Intelligence, 27:267-298, 1999.

[5] A. Ortony, G. Clore, and A. Collins. The Cognitive Structure of Emotions. Cambridge University Press, UK, 1998.

[6] P. Salovey and J. Mayer. Imagination, Cognition and Personality, chapter Emotional Intelligence, pages $185-211$. 1990. 